\title{
Retraction
}

\section{Retracted: The Beneficial Effect of Direct Peritoneal Resuscitation on Septic Shock in Rats}

\author{
BioMed Research International \\ Received 10 May 2015; Accepted 10 May 2015 \\ Copyright (๑ 2015 BioMed Research International. This is an open access article distributed under the Creative Commons \\ Attribution License, which permits unrestricted use, distribution, and reproduction in any medium, provided the original work is \\ properly cited.
}

The paper titled "The beneficial effect of direct peritoneal resuscitation on septic shock in rats" [1], published in BioMed Research International (formerly titled Journal of Biomedicine and Biotechnology), has been retracted as it was found to contain a substantial amount of material from following published article: "Direct peritoneal resuscitation as adjunct to conventional resuscitation from hemorrhagic shock: a better outcome," Surgery, vol. 136, no. 4, pp. 900-908, 2004.

\section{References}

[1] X. Luo, D. Jian, and Z. Lv, "The beneficial effect of direct peritoneal resuscitation on septic shock in rats," Journal of Biomedicine and Biotechnology, vol. 2011, Article ID 743763, 7 pages, 2011. 


\title{
The Beneficial Effect of Direct Peritoneal Resuscitation on
} Septic Shock in Rats

\author{
Xingjun Luo, Daolin Jian, and Zuojun Lv \\ Department of Anesthesiology, Renhe Hospital of Three Gorge University, Yichang 443001, China \\ Correspondence should be addressed to Daolin Jian, rhjdl@ctgu.edu.cn
}

Received 22 July 2011; Accepted 24 August 2011

Academic Editor: Saulius Butenas

Copyright ( 2011 Xingjun Luo et al. This is an open access article distributed under the Creative Commons Attribution License, which permits unrestricted use, distribution, and reproduction in any medium, provided the original work is properly cited.

The high mortality associated with conventionally resuscitated septic shock and the subsequent multiple-organ failure remain a very significant and costly clinical problem. Conventional simple intravenous resuscitation (CR) from septic shock often fails to restore the progressive splanchnic vasoconstriction and hypoperfusion, and fails to reverse gut-derived systemic inflammatory response and fluid sequestration. Numerous interventions have been used to protect organ systems and cellular viability from the lethal injury accompanying hypoperfusion and ischemia but none of these efforts have been sufficient to halt or reverse the main course of the pathophysiology noted with conventional resuscitated shock. Recently, some studies have found that in hemorrhagic shock, direct peritoneal resuscitation (DPR) not only produces sustained hyperperfusion in viscera but also has immunomodulatory and anti-fluid sequestration effects. Although the etiology and pathogenesis of septic shock and hemorrhagic shock differ, both kinds of shock result in hypoperfusion of the intestines and other internal organs. In this paper, we seek to determine whether DPR has a similar therapeutic effect on septic shock/resuscitation.

\section{Introduction}

The high mortality associated with conventionally resuscitated septic shock and the subsequent multipleorgan failure remain a very significant and costly clinical problem [1]. The current treatment for patients with septic shock consists of rapid correction of the vascular deficit. However, studies have found that simple correction of the volume deficit does not fully restore tissue perfusion, despite the provision of overtly adequate volume resuscitation [2]. There are still major alterations in organ microcirculation and tissue metabolism associated with the genesis of an exaggerated gut-derived systemic inflammatory response and a massive fluid shift. Numerous interventions have been used to protect organ systems and cellular viability from the lethal injury accompanying hypoperfusion and ischemia. Some measures have been directed to improve perfusion, whereas others have attempted to enhance the metabolic processes or have used specific antagonists or synthesis inhibitors to modify the state of shock [3-8]. Although blockade of one mediator might provide some protection or give insight into its role in the pathophysiology of shock, none of these efforts have been sufficient to halt or reverse the main course of the pathophysiology noted with conventional resuscitated shock. Thus, the issue of an overall therapy that modifies the pathophysiological process in septic shock/resuscitation remains to be resolved.

Recently, some studies have shown that hemorrhagic shock/resuscitation-mediated intestinal microvascular vasoconstriction and hypoperfusion can be reversed using direct peritoneal resuscitation (DPR), regardless of the timing of $\operatorname{DPR}[9,10]$. This technique uses a clinical peritoneal dialysis solution. Initiation of DPR as adjunct to conventional resuscitation from hemorrhagic shock produces an instant and sustained vasodilation and hyperperfusion of the gut. Furthermore, this splanchnic and distal hyperperfusion occurs without adverse effects on hemodynamics. In addition, the studies also found that DPR has significant therapeutic potential in attenuating the systemic inflammatory response and fluid sequestration associated with CR from hemorrhagic shock [11]. The etiology and pathogenesis of septic shock and hemorrhagic shock differ; however, both kinds 
of shock result in hypoperfusion of the intestines and other internal organs [2, 12-19]. Therefore, we propose that DPR has a similar therapeutic effect on septic shock/resuscitation. This study was designed to evaluate the therapeutic potential of DPR on hemodynamic parameters, the systemic inflammatory response, and the fluid sequestration associated with CR from septic shock.

\section{Materials and Methods}

The research protocol complied with the regulations regarding animal care as published by the Chinese Ministry of Science and Technology and was approved by the Institutional Animal Use and Care Committee of China Three Gorges University. Adult male Sprague-Dawley rats weighing $200 \pm$ $25 \mathrm{~g}$ were purchased from the Experimental Animal Center of Wuhan University and were maintained at the Animal Research Center of China Three Gorges University with a $12 \mathrm{~h}$ light-dark cycle and free access to standard laboratory rat food and water.

2.1. Surgical Preparation. All animals and experimental interventions were performed under aseptic conditions. Anesthesia was induced using $2 \%$ urethane $(1.2 \mathrm{~g} / \mathrm{kg})$ intraperitoneal injection, and supplemental subcutaneous injections ( $25 \%$ the original dose) were given as needed to maintain a surgical plane of anesthesia throughout the experimental protocol. The room temperature was controlled at $26^{\circ} \mathrm{C}$. Surgery was carried out after loss of the blink and withdrawal reflexes. The left carotid artery and right jugular vein were isolated by dissection and were cannulated with PE-50 catheters. The arterial catheter was used for blood sampling and continuous monitoring of arterial pressure. The venous catheter was used for administration of LPS and fluid resuscitation.

2.2. Experimental Protocol. Septic shock was achieved using intravenous LPS. Before administration of LPS, the animals were maintained in a steady state, as defined by stable MAP for at least $30 \mathrm{~min}$. Arterial pressure was measured continuously and was recorded in real time. At $T=0 \mathrm{~min}$, Escherichia coli LPS (serotype O111:B4; Sigma, St Louis, $\mathrm{MO} ; 15 \mathrm{mg} / \mathrm{kg}$ ) was administered intravenously [20-23]. We used a dose that was slightly lower than that of the study by Venkataraman et al. [23] with the goal of increasing survival time and, thus, observation time. When the MAP decreased to be lower than $60 \mathrm{mmHg}$ or the decrease from baseline was not less than $40 \mathrm{mmHg}$, the rats were randomly assigned to one of three treatment groups ( $n=12$ each), which were designated CR, IPS, and DPR. Animals in all groups were resuscitated rapidly with $25 \mathrm{~mL} / \mathrm{kg}$ compound sodium lactate solution infused intravenously from an infusion pump for 30 minutes, and this intervention has been shown to convert classic LPS-induced hypodynamic shock to hyperdynamic shock that more closely resembles human sepsis $[24,25]$. No further intervention was provided after this point in CR group. The IPS group was followed by an intraperitoneal injection of $0.9 \%$ saline $(100 \mathrm{~mL} / \mathrm{kg})$ and the DPR group received an intraperitoneal injection of $2.5 \%$ low calcium peritoneal dialysis solution $(100 \mathrm{~mL} / \mathrm{kg})$. The MAPs of all rats were continuously monitored. Blood samples for measurements of circulating concentrations of inflammatory mediators were obtained at baseline (i.e., after surgical preparation, but prior to LPS injection) and at $T=1 \mathrm{~h}, T=2.5 \mathrm{~h}$, and $T=3.5 \mathrm{~h}$ after injection of LPS. Blood samples for measurements of blood gas were obtained at $T=3.5 \mathrm{~h}$ and were immediately inspected with an automatic blood gas analyzer. Blood samples for measurements of inflammatory mediators were collected in iced tubes. The samples were centrifuged (2000 $\mathrm{g}$ for $10 \mathrm{~min}$ ), and the plasma was aspirated and frozen at $-60^{\circ} \mathrm{C}$ until assayed. Tissue samples for measurements of inflammatory mediators and the dry weight to wet weight ratios were harvested 20 hours after resuscitation. Tissue samples for measurements of inflammatory mediators were collected in iced tubes and were frozen at $-60^{\circ} \mathrm{C}$ until assayed.

2.2.1. Experimental Groups. The rats were randomized to one of three experimental groups after the septic shock was induced: the CR, IPS, and DPR groups. The CR group $(n=12)$ received only conventional simple intravenous resuscitation. The IPS group $(n=12)$ received CR in addition to an intraperitoneal injection of $100 \mathrm{~mL} / \mathrm{kg}$ of $0.9 \%$ saline (IPS). The DPR group $(n=12)$ received CR plus DPR with a clinical $2.5 \%$ low calcium peritoneal dialysis solution $100 \mathrm{~mL} / \mathrm{kg}$ (containing $2.5 \mathrm{~g}$ glucose water, $538 \mathrm{mg}$ sodium chloride, $448 \mathrm{mg}$ sodium lactate, $18.3 \mathrm{mg}$ calcium chloride, and $5.1 \mathrm{mg}$ magnesium chloride, with a $\mathrm{pH}$ of 5.2 and an osmolality of $395 \mathrm{mOsm} / \mathrm{L}$ ). The solution was injected intraperitoneally at the end of CR.

2.2.2. Cytokines Assay. The sandwich enzyme-linked immunosorbent assay technique (ELISA) was used, as suggested by the manufacturer, to determine cytokine profiles (IL-6, TNF- $\alpha$ ) in the serum and IL-6 levels in the liver, lung, and small intestine. ELISA kits were purchased from Bio Co., Ltd. Shanghai Xitang (produced by Sigma Corporation). An amount $(1.0 \mathrm{~g})$ of tissue was removed and placed in $10 \mathrm{~mL}$ of saline buffer at $4^{\circ} \mathrm{C}$. Samples were homogenized for 30 seconds, and homogenates were ultracentrifuged at $5000 \mathrm{rpm}$ for 15 minutes at $4^{\circ} \mathrm{C}$. The total amount of cytokines in the supernatants was measured with an enzymelinked immunosorbent assay. Briefly, 96-well plates were coated with coating antibodies (Endogena, Woburn, Mass.). After inhibition of nonspecific binding, prediluted cytokine standard and experiment samples were added for 2 hours at $37^{\circ} \mathrm{C}$; then, biotin-labeled antibodies (Endogena) were added for 1 hour at $37^{\circ} \mathrm{C}$; enzyme conjugate was added for 0.5 hour at $37^{\circ} \mathrm{C}$. Color was developed using the TMB substrate $\left(3,3^{\prime}, 5,5^{\prime}\right.$-tetramethylbenzidine) for 15 minutes and was stopped using $2 \mathrm{~N} \mathrm{H}_{2} \mathrm{SO}_{4}$. Adsorbance was interpreted at $450 \mathrm{~nm}$. The amount of cytokines was determined from a standard curve and expressed as $\mathrm{pg} / \mathrm{mL}$.

2.2.3. Fluid Sequestration. Total tissue water content was assessed from the dry weight to wet weight ratios in the liver, 


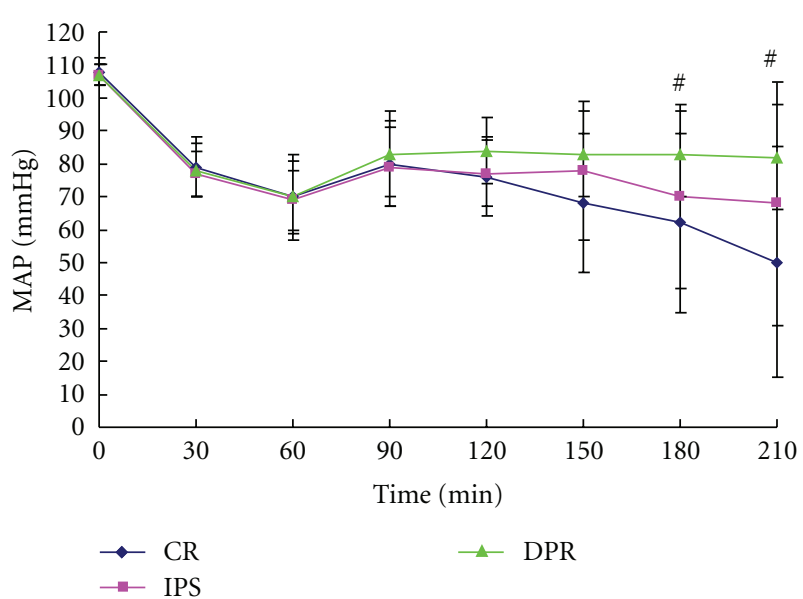

Figure 1: Mean arterial pressure data. ${ }^{\#} P<0.05$ versus CR by oneway analysis of variance and Bonferroni posttest.

small intestine, and lung. Tissue samples of about $10 \mathrm{~g}$ were collected from the 20-hour survivors and dried to a constant weight.

2.3. Statistical Methods. Results are expressed as means \pm SD unless stated otherwise. Differences in survival times between CR, IPS, and DPR were analyzed using the chisquare test. Differences among groups were compared using one-way analysis of variance and the Bonferroni posttest. A result was considered to be significant if the probability of a type-one error was $P$ less than 0.05 .

\section{Results}

3.1. Changes in Mean Arterial Pressure. Animals from the three groups were matched for body weight. There were no significant differences in baseline hemodynamics between the three groups. As expected, septic shock caused a decrease in mean arterial pressure (Figure 1). Resuscitation from septic shock restored mean arterial pressure to near normal levels. Compared with CR and IPS, septic shock mean arterial blood pressure levels were stabilized by DPR after resuscitation (Figure 1).

3.2. Various Arterial Blood Gas Parameters. After the resuscitation, blood lactate concentrations were significantly less in the DPR group compared with CR and IPS at $T=3.5 \mathrm{~h}$. The degree of acid-base imbalance in the DPR groups was significantly lower than in the control groups. There were significant differences in blood $\mathrm{pH}, \mathrm{HCO}_{3}^{-}, \mathrm{BE}(\mathrm{B})$, and BEcef between the DPR and control groups (Table 1).

3.3. Cytokine Profiles. Septic shock and resuscitation caused a significant change in cytokine production. However, the serum and tissue cytokine profiles differed depending on the resuscitation technique. DPR from septic shock was associated with the lowest production of the proinflammatory mediators TNF- $\alpha$ and IL-6 in systemic blood (Figure 2) and
IL-6 in all tissues (Table 2) investigated. This low level of cytokine secretion was associated with a downregulation of the proinflammatory mediators TNF- $\alpha$ and IL- 6 in the tissues and systemic blood. Intraperitoneal infusion of normal saline (IPS) as an adjunct to CR caused less of a decrease in TNF- $\alpha$ and IL- 6 in systemic blood when compared to CR.

3.4. Fluid Sequestration. Compared to the animals in the DPR group, animals in the CR and IPS groups at 20 hours after resuscitation had a lower weight to wet weight ratio in the liver, intestine, and lung, indicating significant edema formation and fluid sequestration in these organs $(P<$ 0.05). In comparison, dry weight to wet weight ratios for these organs in the IPS group were similar to those of the CR group (Table 3). In addition to the failure of fluid mobilization in the CR and IPS groups, which translates into a significant fluid sequestration, $42 \%$ and $25 \%$ of these animals, respectively, died within 20 hours, compared to the $17 \%$ in DPR.

\section{Discussion}

Most visceral organs experience persistent deterioration in blood flow after septic shock with conventional intravenous resuscitation (CR), despite restoration of hemodynamics using aggressive fluid therapy $[2,26]$. This may be related to an obligatory sequestration of fluid in the intracellular and interstitial compartments, especially in the gut and heart [27]. These pathophysiological events result in persistent mucosal ischemia, loss of mucosal integrity, bacterial translocation, and release of proinflammatory/anti-inflammatory mediators, providing overwhelming laboratory and clinical evidence that splanchnic hypoperfusion is a major factor in the systemic inflammatory response after conventionally resuscitated septic shock [28-31]. Recently, some studies have shown that adjunct intraperitoneal resuscitation with clinical peritoneal dialysis solutions enhances splanchnic perfusion and improves blood flow to organs distant from the peritoneal cavity in hemorrhagic shock [32]. In the present study, we demonstrated that adjunct DPR results in hemodynamic stability, reduces the acid-base imbalance, and results in both antifluid sequestration and immunomodulatory effects in a rat model of septic shock after rapid intravenous fluid resuscitation, which translates into improved outcomes.

The primary pathogenesis of septic shock is vascular disorders. In studies of hemorrhagic shock, DPR has been found to have a significant regulatory effect on visceral blood vessel disorders [9-11]. In our study, after rapid intravenous fluid resuscitation, the MAP of all groups was close to $80 \mathrm{mmHg}$. however, the MAP of DPR animals after intravenous resuscitation was more stable than in the other groups, indicating that DPR may play a role in both vascular regulation and rehydration.

We observed that resuscitation of a rat model of septic shock with CR plus DPR instead of CR was associated with lower blood lactate concentrations and lesser acid-base imbalance. It is plausible that resuscitation with additional 
TABLE 1: The various arterial blood gas parameters of the animals in all groups at 3.5 hours. All values represent the mean \pm SD.

\begin{tabular}{|c|c|c|c|c|c|}
\hline Groups & $\mathrm{pH}$ & $\mathrm{HCO}_{3}^{-}(\mathrm{mmol} / \mathrm{L})$ & $\mathrm{BE}(\mathrm{B})(\mathrm{mmol} / \mathrm{L})$ & BEcef $(\mathrm{mmol} / \mathrm{L})$ & $\mathrm{Lac}(\mathrm{mmol} / \mathrm{L})$ \\
\hline $\mathrm{CR}$ & $7.31 \pm 0.05$ & $20.42 \pm 2.16$ & $-5.64 \pm 1.87$ & $-5.91 \pm 2.15$ & $2.24 \pm 0.70$ \\
\hline IPS & $7.28 \pm 0.03$ & $18.30 \pm 3.06$ & $-7.85 \pm 2.61^{*}$ & $-8.47 \pm 3.02^{*}$ & $2.30 \pm 0.63$ \\
\hline DPR & $7.39 \pm 0.05^{\# \text { 记 }}$ & $23.64 \pm 2.03^{* \text { 访 }}$ & $-1.21 \pm 1.68^{\# \mathcal{L}_{3}}$ & $-1.28 \pm 1.92^{\text {\#记 }}$ & $1.52 \pm 0.31^{\text {*纺 }}$ \\
\hline
\end{tabular}

${ }^{*} P<0.05,{ }^{\#} P<0.01$ versus CR by one-way analysis of variance and Bonferroni posttest. ${ }^{\triangle} P<0.05$, iै $P<0.01$ versus IPS by one-way analysis of variance and Bonferroni posttest.

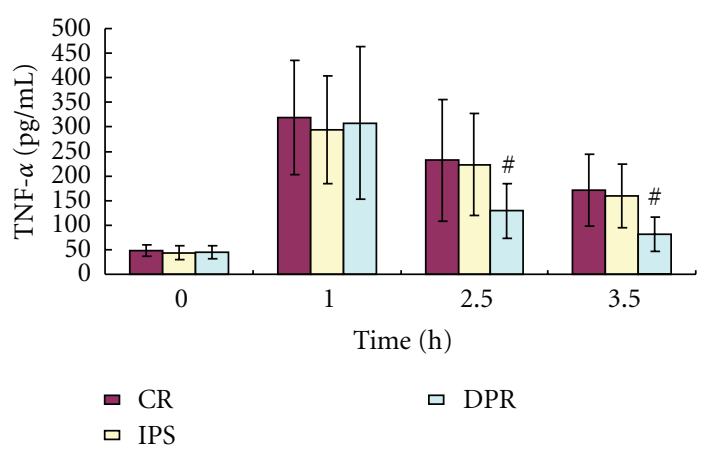

(a)

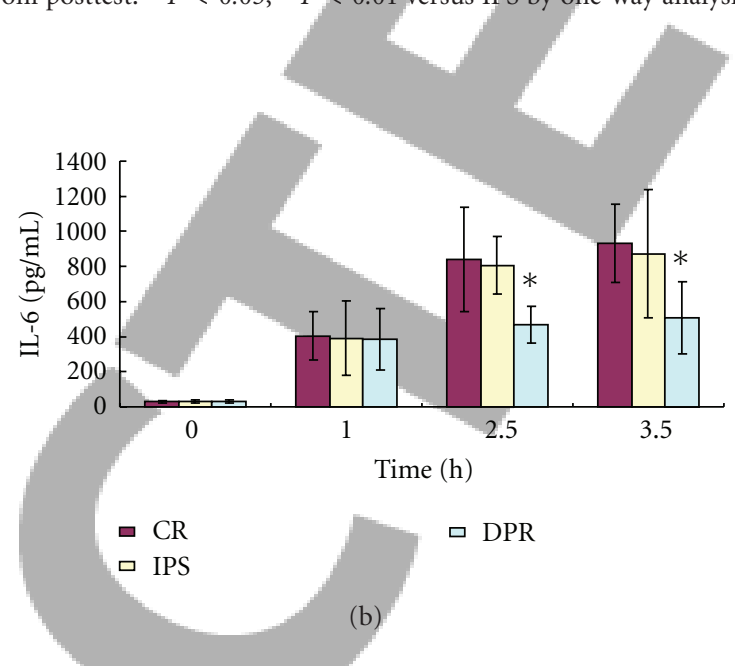

FIGURE 2: Comparison of plasma concentrations of TNF- $\alpha$ (a), IL-6 (b) in septic rats after different resuscitation methods. ${ }^{\#} P<0.05$ versus CR and IPS by one-way analysis of variance and Bonferroni posttest. ${ }^{*} P<0.01$ versus CR and IPS by one-way analysis of variance and Bonferroni posttest.

DPR may be associated with lower lactate levels secondary to improved tissue perfusion. Increasingly, however, it is becoming apparent that hyperlactatemia in sepsis or endotoxemia is less a reflection of impaired oxygen delivery than a profound alteration in intermediary metabolism that favors a marked increase in glucose-to-lactate flux, independent of tissue oxygenation [33]. Therefore, it is conceivable that the anti-inflammatory effects of DPR modulated LPS-induced aerobic glycolysis in various cell types, thereby reducing the circulating levels of lactate.

The body's immune response to noxious stimuli can lead to tissue damage and organ dysfunction. The degree of activation of this response depends on the balance of proinflammatory cytokines, such as IL- 6 , and TNF- $\alpha$, and the cytokine secretion inhibitory factor. TNF- $\alpha$, IL- 6 and other inflammatory cytokines are involved in septic shock and inflammation after resuscitation and play an important role in the subsequent progression of the disease. In particular, TNF- $\alpha$ was recognized as a primary mediator of endotoxin shock and multiple organ dysfunction [34]. IL-6 is a cytokine that is synthesized and secreted by various phagocytes after stimulation and plays a variety of biological roles. It can cause shock to advance from reversible to irreversible and has a significant correlation with the prognosis of the patient. There is a significant correlation between plasma IL-6 levels, the function of important organs, and the survival of animals [35]. In the present study, it is likely that DPR exerted its maximal downregulation effect on TNF- $\alpha$ and IL- 6 during their peak elevation in the early phase after CR.
The tissue dry weight to wet weight ratio reflects the degree of fluid sequestration. After septic shock, liquid transfers between the different organizational compartments. The water was isolated inside the cell and the tissue space, causing clinical tissue edema. We found in experiment that the animals in the DPR group had higher tissue dry/wet weight ratios. The mechanisms that allow DPR to prevent or reverse fluid sequestration are related to the osmotic stress produced by the intraperitoneal solution (Lactate-G2.5\%) that is used for DPR. Under normal physiologic conditions, fluid flow across the capillary wall is determined by the capillary hydraulic permeability and the transcapillary hydrostatic and oncotic pressures (Starling forces). The imbalance in the Starling forces favors a slight continuous fluid filtration from the vascular space, which is balanced by an equal interstitial fluid volume outflow through lymphatics; therefore, the interstitial fluid volume and pressure are kept constant. DPR adds a filtration force to the transcapillary Starling forces and creates a crystalloid osmotic gradient. Under conditions of crystalloid osmotic transient, if only $1.5 \%$ to $2 \%$ of the capillary hydraulic permeability is accounted for by transcellular water-exclusive pathways (Aquaporin-1), then 50\% of the osmotic water flow occurs through these Aquaporin water channels, whereas the other half occurs through paracellular pathways [36, 37]. The osmotic-driven ultrafiltrate is not exclusively derived from the vascular volume; however, a larger fraction is derived from the cellular water of all tissues bordering the peritoneal cavity $[38,39]$, as hyperosmolality is a major factor in the mechanisms of cell volume regulation 
TABLE 2: Visceral tissue concentrations of cytokines IL-6. All values represent the mean \pm SD.

\begin{tabular}{lccc}
\hline Groups & Liver $(\mathrm{pg} / \mathrm{mL})$ & Ileum $(\mathrm{pg} / \mathrm{mL})$ & Lung $(\mathrm{pg} / \mathrm{mL})$ \\
\hline CR & $2740 \pm 192$ & $647 \pm 204$ & $610 \pm 140$ \\
IPS & $2589 \pm 363$ & $507 \pm 230$ & $476 \pm 170$ \\
DPR & $2230 \pm 245^{\# \Delta}$ & $230 \pm 121^{\# \Delta}$ & $274 \pm 93^{\# \Delta}$ \\
\hline
\end{tabular}

${ }^{*} P<0.05,{ }^{\#} P<0.01$ versus CR by one-way analysis of variance and Bonferroni posttest. ${ }^{\triangle} P<0.05$, iै $P<0.01$ versus IPS by one-way analysis of variance and Bonferroni posttest.

TABLE 3: Visceral tissue wet/dry weight ratios. All values represent the mean $\pm \mathrm{SD}$.

\begin{tabular}{lccc}
\hline Groups & Liver $(\%)$ & Intestines $(\%)$ & Lung $(\%)$ \\
\hline CR & $30.0 \pm 2.2$ & $23.0 \pm 2.2$ & $21.5 \pm 1.3$ \\
IPS & $29.3 \pm 2.5$ & $22.2 \pm 2.5$ & $20.9 \pm 0.9$ \\
DPR & $32.0 \pm 1.3^{* \Delta}$ & $25.3 \pm 1.7^{* \text { 出 }}$ & $22.8 \pm 1.5^{* \Delta}$ \\
\hline${ }^{*}$
\end{tabular}

${ }^{*} P<0.05,{ }^{\#} P<0.01$ versus CR by one-way analysis of variance and Bonferroni posttest. ${ }^{\triangle} P<0.05$, ${ }^{\natural} P<0.01$ versus IPS by one-way analysis of variance and Bonferroni posttest.

acting through modulation of transcellular ionic exchange $[33,40]$. In addition to an osmotic effect, the intraperitoneal Lactate-G2.5\% changes the intraperitoneal hydrostatic pressure in a nonlinear fashion depending on the instilled volume $[39,41]$. These intraperitoneal forces cause simultaneous osmotic-driven water flux into the peritoneal cavity and, in the opposite direction, a subordinate hydrostatic-driven fluid convection into tissues bordering the peritoneal cavity, especially the abdominal muscle. Although the osmotic water flow is limited by dissipation of the osmotic gradient due to glucose absorption, the hydrostatic-driven water flow promotes tissue hydration, restores lymph flow, and constitutes a slow resuscitation compartment, which expands the vascular volume over time [11].

The redistribution of blood in septic shock-induced microvascular impairment together with a systemic inflammatory response results in a redistribution of blood flow between and within vital organ systems. The redistribution of blood flow results in the peripheral microcirculation insufficiency. In particular, shock causes low capillary blood flow via reduction in perfusion pressure, edema of the endothelial lining, and subsequent plugging of capillaries by activated leukocytes. The immediate effect of these capillary events is a reduction in the number of perfused capillaries and deregulation of the capillary Starling forces governing the basic capillary function of the transcapillary fluid exchange. Intravascular $\mathrm{CR}$ is intended to rapidly restore intravascular volume and is considered adequate when central hemodynamics are restored to normal levels. However, CR from septic shock often fails to correct the multifaceted pathophysiologic capillary perfusion/functional deficits of the shock syndrome [11]. Furthermore, CR correlates with the time at which reperfusion injury occurs [42]. Although ischemia of tissues determines the extent of reperfusion injury, it is becoming increasingly evident that this injury occurs and is related to the immune system activation during conventional intravascular fluid resuscitation $[43,44]$. This activation elicits an exaggerated systemic inflammatory response, which affects neutrophils and, thus, the microcirculation, especially those of the visceral organs.
Recently, Hopkins et al. [45] used peritoneal infusions of $2.5 \%$ dextrose solution as an adjunct to resuscitation of two very-low-birth-weight infants having perforated necrotizing enterocolitis. In the report, dextrose appeared to control the shock state to allow for general anesthesia and major surgical resection. This is similar to our experimental results. In view of the positive effects of DPR on septic shock in both our study and Hopkins' clinical report, we propose that, under the appropriate conditions, a cautious clinical trial is feasible. However, although our study proved that DPR may have some positive effects on resuscitation in septic shock, it was only preliminary and did not further explore the optimal method and dose of DPR, the effect difference of DPR in different resuscitation conditions and the mechanism of DPR in septic shock, and so forth, all of which were worthy of further study to provide more research supports for this new method in septic shock.

\section{Conclusion}

In conclusion, DPR is a new resuscitation technique that is conceptually different from either the conventional crystalloid resuscitation or the low-volume intravascular hypertonic saline resuscitation. DPR uses a balanced salt solution containing glucose as an osmotic agent. This clinical solution is administered intraperitoneally at the time of CR completion. The present study demonstrates that DPR as an adjunct to CR has beneficial effects on the pathophysiology of septic shock, including stabilizations in hemodynamic parameters, reductions in acid-base imbalances, immunomodulation, and decreased fluid sequestration. With further research, we believe that adjunct DPR may play an increasingly important role in the future management of septic shock.

\section{Conflict of Interests}

The authors declare that they have no conflict of interest. 


\section{Acknowledgment}

The authors would like to thank Changing Xiang for providing great support to their study.

\section{References}

[1] H. T. Hassoun, B. C. Kone, D. W. Mercer, F. G. Moody, N. W. Weisbrodt, and F. A. Moore, "Post-injury multiple organ failure: the role of the gut," Shock, vol. 15, no. 1, pp. 1-10, 2001.

[2] L. B. Hiltebrand, V. Krejci, A. Banic, D. Erni, A. M. Wheatley, and G. H. Sigurdsson, "Dynamic study of the distribution of microcirculatory blood flow in multiple splanchnic organs in septic shock," Critical Care Medicine, vol. 28, no. 9, pp. 32333241, 2000.

[3] B. Dahlbäck, "Progress in the understanding of the protein C anticoagulant pathway," International Journal of Hematology, vol. 79, no. 2, pp. 109-116, 2004.

[4] S. J. Fitch and J. R. Gossage, "Optimal management of septic shock: rapid recognition and institution of therapy are crucial," Postgraduate Medicine, vol. 111, no. 3, pp. 53-66, 2002.

[5] B. D. Freeman and C. Natanson, "Anti-inflammatory therapies in sepsis and septic shock," Expert Opinion on Investigational Drugs, vol. 9, no. 7, pp. 1651-1663, 2000.

[6] C. L. Holmes, B. M. Patel, J. A. Russell, and K. R. Walley, "Physiology of vasopressin relevant to management of septic shock," Chest, vol. 120, no. 3, pp. 989-1002, 2001.

[7] A. Meier-Hellmann, S. G. Sakka, and K. Reinhart, "Catecholamines and splanchnic perfusion," Schweizerische Medizinische Wochenschrift, vol. 130, no. 50, pp. 1942-1947, 2000.

[8] K. Werdan, "Pathophysiology of septic shock and multiple organ dysfunction syndrome and various therapeutic approaches with special emphasis on immunoglobulins," Therapeutic Apheresis, vol. 5, no. 2, pp. 115-122, 2001.

[9] E. R. Zakaria, R. N. Garrison, T. Kawabe et al., "Direct peritoneal resuscitation from hemorrhagic shock: effect of time delay in therapy initiation," Journal of Trauma, vol. 58, no. 3 , pp. 499-508, 2005.

[10] E. R. Zakaria, R. N. Garrison, D. A. Spain et al., "Intraperitoneal resuscitation improves intestinal blood flow following hemorrhagic shock," Annals of Surgery, vol. 237, no. 5, pp. 704-713, 2003.

[11] R. N. Garrison, A. A. Conn, P. D. Harris, and E. R. Zakaria, "Direct peritoneal resuscitation as adjunct to conventional resuscitation from hemorrhagic shock: a better outcome," Surgery, vol. 136, no. 4, pp. 900-908, 2004.

[12] T. M. Fruchterman, D. A. Spain, M. A. Wilson, P. D. Harris, and R. N. Garrison, "Selective microvascular endothelial cell dysfunction in the small intestine following resuscitated hemorrhagic shock," Shock, vol. 10, no. 6, pp. 417-422, 1998.

[13] R. R. Ivatury, R. J. Simon, S. Islam, A. Fueg, M. Rohman, and W. M. Stahl, "A prospective randomized study of end points of resuscitation after major trauma: global oxygen transport indices versus organ-specific gastric mucosal $\mathrm{pH}$," Journal of the American College of Surgeons, vol. 183, no. 2, pp. 145-154, 1996.

[14] D. LeDoux, M. E. Astiz, C. M. Carpati, and E. C. Rackow, "Effects of perfusion pressure on tissue perfusion in septic shock," Critical Care Medicine, vol. 28, no. 8, pp. 2729-2732, 2000.

[15] P. M. Reilly, K. B. Wilkins, K. C. Fuh, U. Haglund, and G. B. Bulkley, "The mesenteric hemodynamic response to circulatory shock: an overview," Shock, vol. 15, no. 5, pp. 329-343, 2001.
[16] J. P. Revelly, T. Ayuse, N. Brienza, H. E. Fessler, and J. L. Robotham, "Endotoxic shock alters distribution of blood flow within the intestinal wall," Critical Care Medicine, vol. 24, no. 8, pp. 1345-1351, 1996.

[17] E. Ruokonen, J. Takala, A. Kari, H. Saxen, J. Mertsola, and E. J. Hansen, "Regional blood flow and oxygen transport in septic shock," Critical Care Medicine, vol. 21, no. 9, pp. 1296-1303, 1993.

[18] J. Tuchschmidt, J. Fried, M. Astiz, and E. Rackow, "Elevation of cardiac output and oxygen delivery improves outcome in septic shock," Chest, vol. 102, no. 1, pp. 216-220, 1992.

[19] B. Vallet, N. Lund, S. E. Curtis, D. Kelly, and S. M. Cain, "Gut and muscle tissue $\mathrm{PO}_{2}$ in endotoxemic dogs during shock and resuscitation," Journal of Applied Physiology, vol. 76, no. 2, pp. 793-800, 1994.

[20] Y. Hui, J. Du, C. Tang, G. Bin, and H. Jiang, "Changes in arterial hydrogen sulfide $\left(\mathrm{H}_{2} \mathrm{~S}\right)$ content during septic shock and endotoxin shock in rats," Journal of Infection, vol. 47, no. 2, pp. 155-160, 2003.

[21] K. Koike, "Animal models for sepsis," Nippon Rinsho, vol. 62, no. 12, pp. 2262-2266, 2004.

[22] S. J. Parker and P. E. Watkins, "Experimental models of gramnegative sepsis," British Journal of Surgery, vol. 88, no. 1, pp. 22-30, 2001.

[23] R. Venkataraman, J. A. Kellum, M. Song, and M. P. Fink, "Resuscitation with Ringer's ethyl pyruvate solution prolongs survival and modulates plasma cytokine and nitrite/nitrate concentrations in a rat model of lipopolysaccharide-induced shock," Shock, vol. 18, no. 6, pp. 507-512, 2002.

[24] E. A. Deitch, "Animal models of sepsis and shock: a review and lessons learned," Shock, vol. 9, no. 1, pp. 1-11, 1998.

[25] P. E. Wischmeyer, M. Kahana, R. Wolfson, H. Ren, M. M. Musch, and E. B. Chang, "Glutamine induces heat shock protein and protects against endotoxin shock in the rat," Journal of Applied Physiology, vol. 90, no. 6, pp. 2403-2410, 2001.

[26] F. M. van Haren, J. W. Sleigh, P. Pickkers, and J. G. van der Hoeven, "Gastrointestinal perfusion in septic shock," Anaesthesia and Intensive Care, vol. 35, no. 5, pp. 679-694, 2007.

[27] P. F. Moon, M. A. Hollyfield-Gilbert, T. L. Myers, and G. C. Kramer, "Effects of isotonic crystalloid resuscitation on fluid compartments in hemorrhaged rats," Shock, vol. 2, no. 5, pp. 355-361, 1994.

[28] I. Farquhar, C. M. Martin, C. Lam, R. Potter, C. G. Ellis, and W. J. Sibbald, "Decreased capillary density in vivo in bowel mucosa of rats with normotensive sepsis," Journal of Surgical Research, vol. 61, no. 1, pp. 190-196, 1996.

[29] E. E. Moore, F. A. Moore, R. J. Franciose, F. J. Kim, W. L. Biffl, and A. Banerjee, "The postischemic gut serves as a priming bed for circulating neutrophils that provoke multiple organ failure," Journal of Trauma, vol. 37, no. 6, pp. 881-887, 1994.

[30] A. Secchi, R. Wellmann, E. Martin, and H. Schmidt, "Dobutamine maintains intestinal villus blood flow during normotensive endotoxemia: an intravital microscopic study in the rat," Journal of Critical Care, vol. 12, no. 3, pp. 137-141, 1997.

[31] C. J. Theuer, M. A. Wilson, G. D. Steeb, and R. N. Garrison, "Microvascular vasoconstriction and mucosal hypoperfusion of the rat small intestine during bacteremia," Circulatory Shock, vol. 40, no. 1, pp. 61-68, 1993.

[32] E. R. Zakaria, R. T. Hurt, P. J. Matheson, and R. N. Garrison, "A novel method of peritoneal resuscitation improves organ perfusion after hemorrhagic shock," American Journal of Surgery, vol. 186, no. 5, pp. 443-448, 2003.

[33] Y. Miyata, S. Muto, S. Yanagiba, and Y. Asano, "Extracellular $\mathrm{Cl}(-)$ modulates shrinkage-induced activation of $\mathrm{Na}(+) / \mathrm{H}(+)$ 
exchanger in rat mesangial cells," American Journal of Physiology-Cell Physiology, vol. 278, no. 6, pp. C1218-C1229, 2000.

[34] D. W. Loe, R. G. Deeley, and S. P. Cole, "Biology of the multidrug resistance-associated protein, MRP," European Journal of Cancer, vol. 32, no. 6, pp. 945-957, 1996.

[35] A. Spittler, M. Razenberger, H. Kupper et al., "Relationship between interleukin-6 plasma concentration in patients with sepsis, monocyte phenotype, monocyte phagocytic properties, and cytokine production," Clinical Infectious Diseases, vol. 31, no. 6, pp. 1338-1342, 2000.

[36] B. Rippe and G. Stelin, "Simulations of peritoneal solute transport during CAPD. Application of two-pore formalism," Kidney International, vol. 35, no. 5, pp. 1234-1244, 1989.

[37] E. R. Zakaria and B. Rippe, "Osmotic barrier properties of the rat peritoneal membrane," Acta Physiologica Scandinavica, vol. 149, no. 3, pp. 355-364, 1993.

[38] H. Demissachew, J. Lofthouse, and M. F. Flessner, "Tissue sources and blood flow limitations of osmotic water transport across the peritoneum," Journal of the American Society of Nephrology, vol. 10, no. 2, pp. 347-353, 1999.

[39] E. R. Zakaria, J. Lofthouse, and M. F. Flessner, "Effect of intraperitoneal pressures on tissue water of the abdominal muscle," American Journal of Physiology-Renal Physiology, vol. 278, no. 6, pp. F875-F885, 2000.

[40] A. Breborowicz, A. Polubinska, and D. G. Oreopoulos, "Changes in volume of peritoneal mesothelial cells exposed to osmotic stress," Peritoneal Dialysis International, vol. 19, no. 2, pp. 119-123, 1999.

[41] E. R. Zakaria, J. Lofthouse, and M. F. Flessner, "Hydrostatic and osmotic pressures modulate partitioning of tissue water in abdominal muscle during dialysis," Peritoneal Dialysis International, vol. 19, supplement 2, pp. S208-S211, 1999.

[42] K. Waxman, "Shock: ischemia, reperfusion, and inflammation," New Horizons, vol. 4, no. 2, pp. 153-160, 1996.

[43] P. Rhee, D. Wang, P. Ruff et al., "Human neutrophil activation and increased adhesion by various resuscitation fluids," Critical Care Medicine, vol. 28, no. 1, pp. 74-78, 2000.

[44] K. Waxman, "What mediates tissue injury after shock?" New Horizons, vol. 4, no. 2, pp. 151-152, 1996.

[45] J. W. Hopkins, B. Chandramouli, and P. Wall, "Preliminary resuscitation for perforated necrotizing enterocolitis: 2 cases treated with initial direct peritoneal resuscitation," Journal of Pediatric Surgery, vol. 46, no. 1, pp. 237-240, 2011.
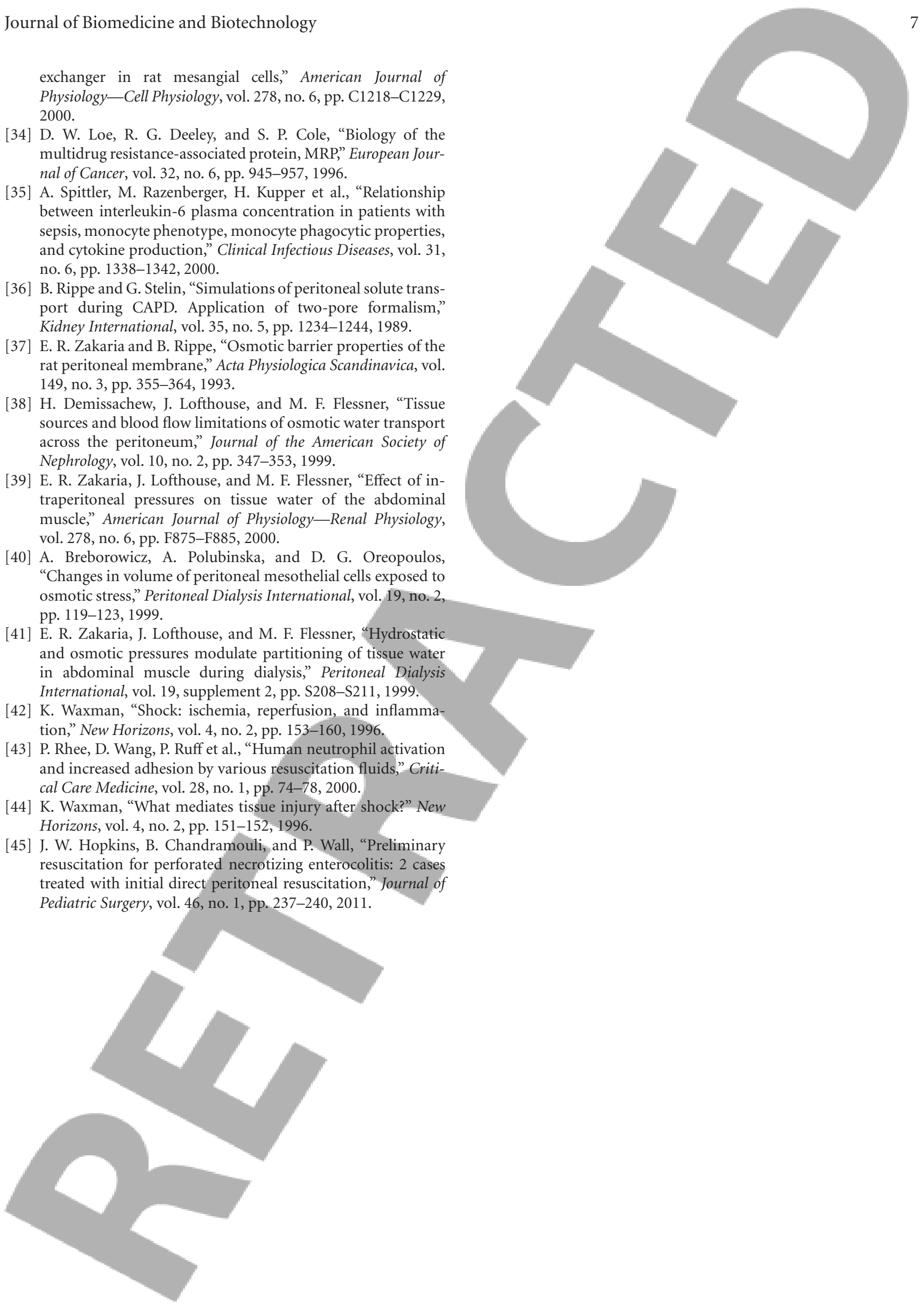